\title{
An Efficient Four Channels 3D Plasmonic Demultiplexer
}

\author{
Mohammed Nadhim Abbas \\ Electrical Engineering Department \\ College of Engineering, University of Baghdad \\ Baghdad, Iraq
}

\author{
Ahmed Abdulredha Ali \\ Electrical Engineering Department \\ College of Engineering, University of Baghdad \\ Baghdad, Iraq
}

\begin{abstract}
The four channels of 3D plasmonic demultiplexer structure are selective based on a nanocavity that proposed and numerically simulated by using the finite element method by using COMSOL4.4 software package The required filtered wavelength can be investigated by selecting an appropriate length of the nanocavity and refractive index of dielectric that filled nanocavity. The selecting wavelength of for 3D channels are dependent on three geometric parameters thickness, width and length. Four output channels structure based on four perpendicular nanocavities that proposed to design a subwavelength plasmonic splitter, and demultiplexer. 3D plasmonic demultiplexer with $1 \times \quad 4$ channels it's peak transmisson of four channels occurs at around the wavelengths of $810 \mathrm{~nm}, 990 \mathrm{~nm}, 1210 \mathrm{~nm}$ and $1500 \mathrm{~nm}$, with transmittance effeceincy are $57 \%, 72 \%, 74 \%$, $70 \%$ respectively.Three materials used to build structure ,metal used as a silver and two types of dielectric quartz with refractive index 1.5 and air with refractive index 1.
\end{abstract}

\section{General Terms}

3D WDM structure , 3D plasmonic (WDM), 3D waveguide, Demultiplexer

\section{Keywords}

Plasmonics, Surface plasmon polration, 3D nanocavity waveguide, resonance wavelength, 3D plasmonic demultiplexer.

\section{INTRODUCTION}

Surface plasmon polaritons (SPPs) are waves propagated in the interface between the metal and dielectric, result from the interplay between the free electrons in metal and electromagnetic field which is incident on dielectric, and decaying exponentially in the direction perpendicular to the interface $[1,2]$. Surface plasmon polaritons have very good promising applications in the high density of optical component for integrated optical circuits and devices due to their removing of diffraction limit and light manipulation on subwavelength scales $[1,3]$. Today, the devices based on Surface plasmon polaritons, suchresonator as the all-optical switching, filtterings [4-6], Mach-Zehnder interferometers [7], splitter [8], modulator [9], mirror [10], sensor [11], and Bragg reflectors [12,13] are simulated numerically and demonstrated experimentally. Bragg reflectors are fabricated by instead of glue two kinds of dielectrics in metalinsulatormetal (MIM) or insulator-metal-insulator (IMI) plasmonic waveguides [12], and can be achieved and filtration functions. IMI waveguide leads to less loss of distance longer propagation, but fails to confine the light in subwavelength scale, and therefore, is not suitable for high optical integration [14]. The MIM waveguide has a strong confinement of the light and acceptable diffusion length Surface plasmon polaritons [12]. Great lately, and some simple filters suggested wavelength plasmonic guide, such as plasmonic waveguide filters on the teeth form [15,16], drop channel filters with resonators disk [17], rectangular geometric resonators [2.18], and ring resonators [17.19]. They overcome the complexity of the manufacturing Bragg reflectors and reduce the length of the postponement for Surface plasmon polaritons. There are two types of filters in plasmonic waveguide MIM, any band pass and band-stop filters. Candidate band-stop prohibits the light with a specific frequency through the publication of the waveguide structures [15.18]. Band pass filter, which allows the transfer of certain frequency of light, is also very important in nanoscale optical devices [16.19]. Some devices, such as Surface plasmon polaritons reflectors need band gaps wide [13]. In many cases, such as wavelength division multiplexing systems (WDM), however, and desired bandwidth narrow bandwidth [16.20]

In this paper,has been proposed a novel and simple 3D plasmonic demultiplexer on the basis of MIM waveguide resonator with nanocavity (any naocavity in the form of rectangular). Has been focus on Tunable filter of this structure candidate Properties. The characteristics of the wavelength shift Resonant modes in nanocavity and calculated on the basis of resonant naocavity rectangular-shaped theory .It is ratified by a limited, Finite Element (FEM) method [21]. Another result obtained Using COMSOL 4.4 software multiphsics simulation shows that the bandwidth of the resonance spectra can be Manipulated by nanocavity after coupling between the waveguide, which is Exact analysis of the theory side of a road time.

\section{STRUCTURE AND THEORETIES}

This section will explain how to design the structure of a 3D wavelength division demultiplexer based on plasmonic resonators (WDM).The proposed structure for the 3D wavelength division demultiplexing is consist from four channels ,However, the structure contain one input and four outputs. The locations of ports placed in the exit of each drop channels and one of ports placed in started of main bus plasmonic waveguide. The function of ports are to detect powers in outputs and input.The proposed structure based on plasmonic resonators ,the nanocavity consider resonator for selecting resonance wavelength dependent on geometic parameters of nanocavity as length , width and thickness on resonators ,the figure (1) show the structure of two channels 3D wavelength division demultiplexing. The two channels located above of main bus plasmonic waveguide and connected to main bus by nanocavity. The structure consist of metal as silver and insulator as quartz and forming plasmonic waveguide silver-insulator-silver. The main bus waveguide and drop channels filled by an air as insulator with refractive index 1 and the nanocavity will fill by quartz as insulator with refractive index 1.5.The dielectric constant of metal (silver) is frequency dependent. In this paper used experimental data and a more detailed fit of the data reported by Johnson and Christy for silver that gives more accuracy from Drude model 
[22].The incident light should be polarized (TM) because of the TE polarized light cannot confinement in nanoscale width of resonator but the TM waves can propagated at subwavelength scale resonator. In 3D plasmonic (WDM) the principle of selections wavelengths are the same that in the 2D plasmonic (WDM). The resonators shape has effect on filter wavelength, also dependent on the refractive index dielectric filled the resonator. The region of connection resonators with bus waveguide it's role to select resonance wavelength .

The effective refractive index of Surface plasmon polaritons is related with the wavelength $\lambda$ and the width $w$ of bus waveguide.Their dispersion relation is governed by [23].

$$
\varepsilon_{i n} K_{y 2}+\varepsilon_{m} K_{y 1} \tanh \left(-\frac{i K_{y 1}}{2} w\right)=0
$$

$K_{y 1}$ and $K_{y 2}$ defined as $K_{y 1}^{2}=\varepsilon_{i n} K_{0}^{2}-\beta^{2}$ and $K_{y 2}^{2}=\varepsilon_{m} K_{0}^{2}-\beta^{2}$ where $\varepsilon_{i n}$ and $\varepsilon_{m}$ are respectively dielectric constants of the insulator and the metal. $K_{0}=$ $2 \pi / \lambda$ is the wave number of light with wavelength $\lambda$ in vacuum. The effective refractive index is represented $n_{\text {eff }}=$ $\beta_{R} / K_{0}$.

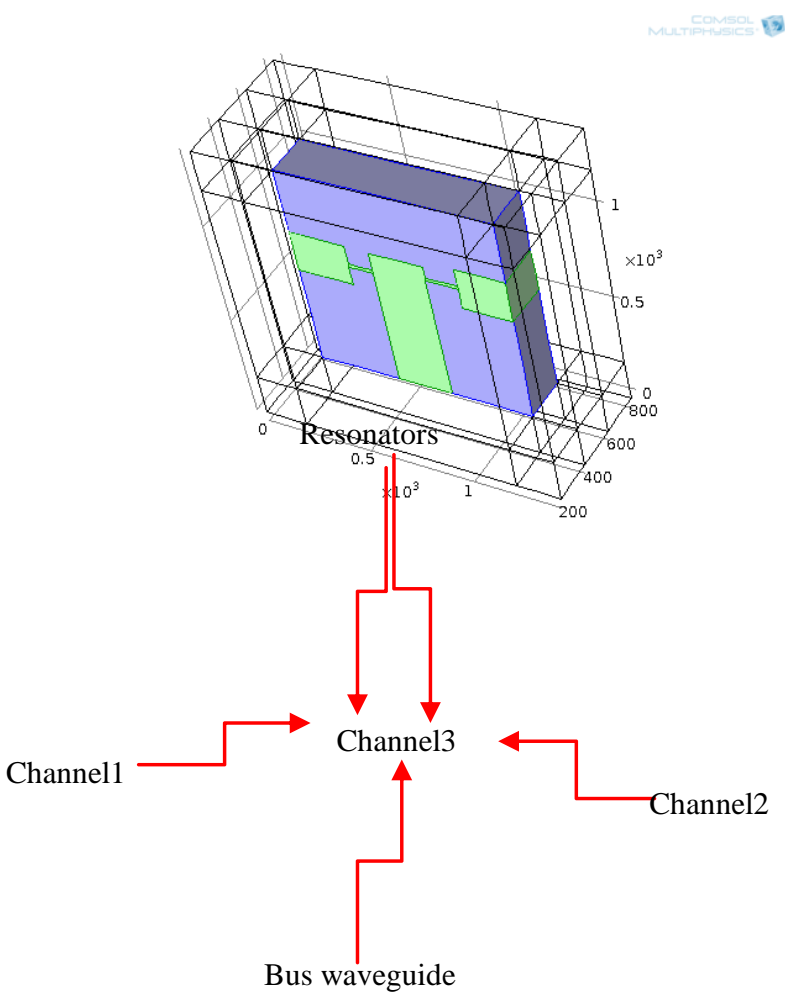

Fig1: Shows 3D plasmonic demultiplexer with upper two channels

The two channels in the lower bus waveguide will select center wavelength about in another part of the spectrum. Figure (2) 3D plasmonic demultiplexer with two channels connected to the lower of bus waveguide

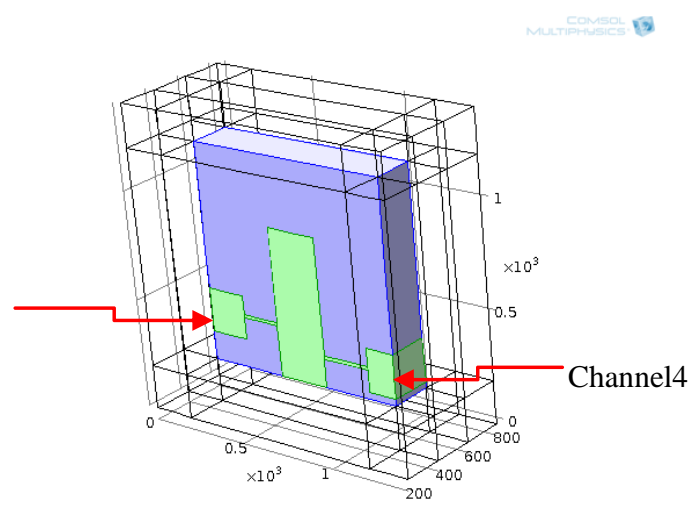

Fig2: Shows 3D plasmonic demultiplexer with lower two channels

\section{PERFECTLY MATCHED LAYER} (PML)

PML, while it has revolutionized absorbing boundaries for wave equations, especially (but not limited to) the electromagnetic. PML is a reflection of just less if it has been solving equations wave exactly. PML still waves absorbing material that spread inside still attenuated, even separate waves. The border between the PML and the normal medium is no longer a reflection less, but because the small reflections individualize is (presumably) a good approximation to the exact wave .The figure (3) shows the perfectly matched layer (PML) from side view of demultiplexer structure. The figure (4) shows the perfectly matched layer (PML) from front view of demultiplexer structure.

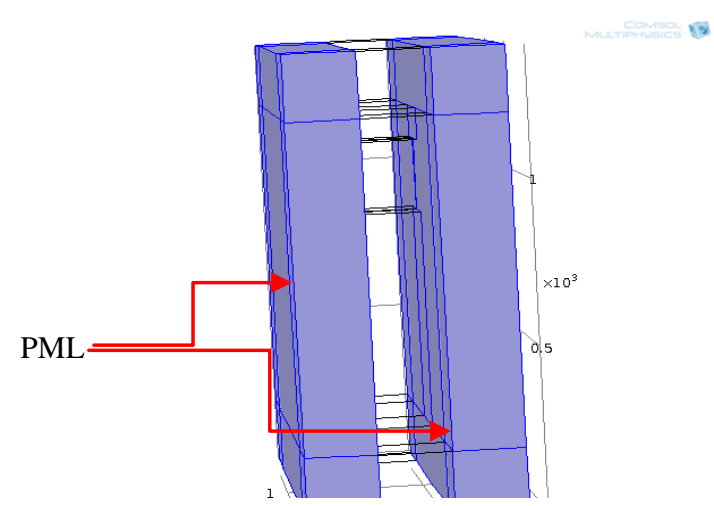

Fig3: Schematic diagram demultiplexer structure of side view shows (PML) 


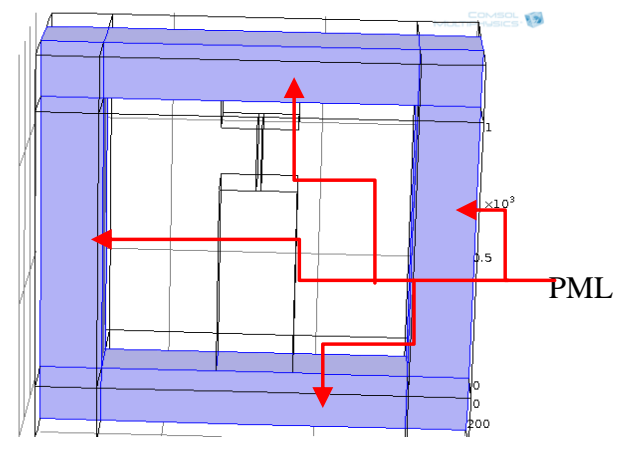

Fig4: Schematic diagram demultiplexer structure of front view shows (PML)

In the figures $(1,2)$ collar blue represent the metal of structure and the green collar represent insulator (dielectric) of structure 3D plasmonic waveguide. In the figures $(3,4)$ collar blue represent matched perfect layer.

\section{SIMULATION AND RESULT}

The results of 3D structure are like the results of 2D structure ,the flowing results will show the trnsmission efficeincy and reflectivty for 3D plasmonic (WDM) structure. The thickness of resonators effect on the shiffted of wavelength channel in addition other parameters that effected in the result of $2 \mathrm{D}$ structure . Figure (5) shows transmission spectrum of two output channels for two channels that connected to upper the bus plasmonic waveguide.

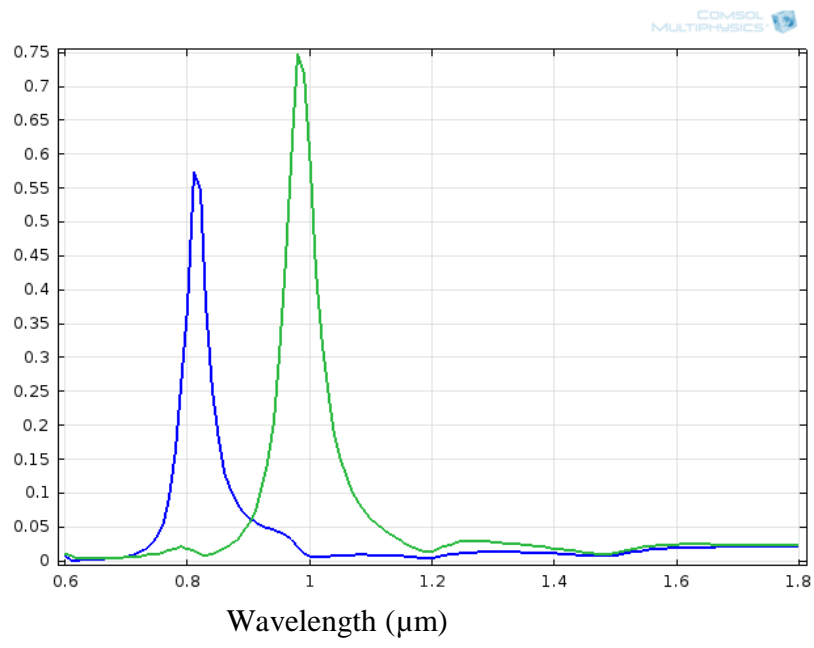

Fig5: Transmission spectra of two output channels of 3D plasmonic demultiplexer structure.

One can see that the peak wavelength of first channel occurs at around the wavelength of $810 \mathrm{~nm}$ and its transmittance is about $57 \%$. The peak wavelength of second channel occurs at around the wavelength of $990 \mathrm{~nm}$ and its transmittance is about $72 \%$. Figure (6) shows transmission spectrum of two output channels for two channels that connected to lower of the bus waveguide. One can be note the peak transmission of two lower channels located at around the wavelength of $1210 \mathrm{~nm}$ and $1500 \mathrm{~nm}$ its transmittance is about $74 \%$ and $70 \%$ respectively. 3D plasmonic demultiplexer with $1 \times 4$ channels it's peak transmisson of four channels occurs at around the wavelengths of $810 \mathrm{~nm}, 990 \mathrm{~nm}, 1210 \mathrm{~nm}$ and $1500 \mathrm{~nm}$, with transmittance effeceincy are $57 \%, 72 \%, 74 \%, 70 \%$ respectively. Figure (7)shows transmission spectrum of four output channels

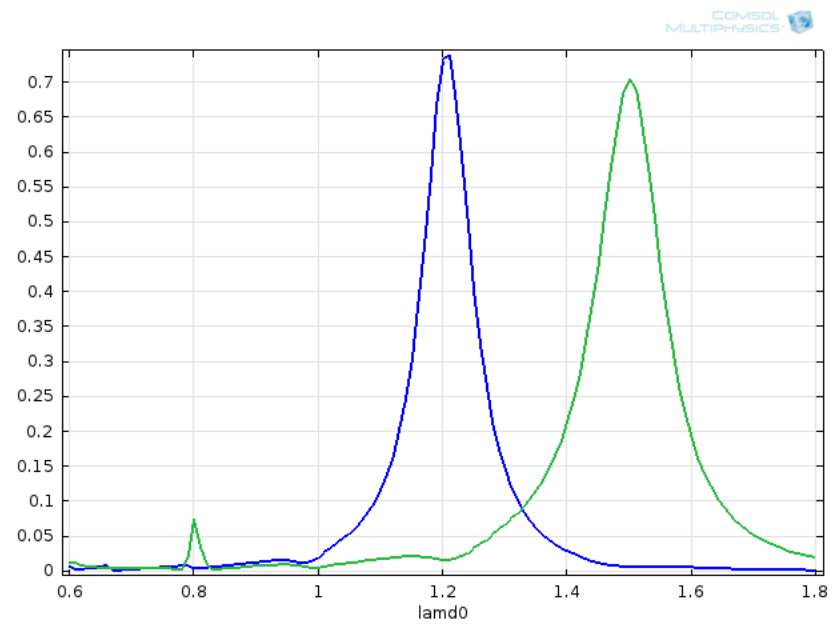

Wavelength (um)

Fig6: Transmission spectra of two output channels of 3D plasmonic demultiplexer structure.

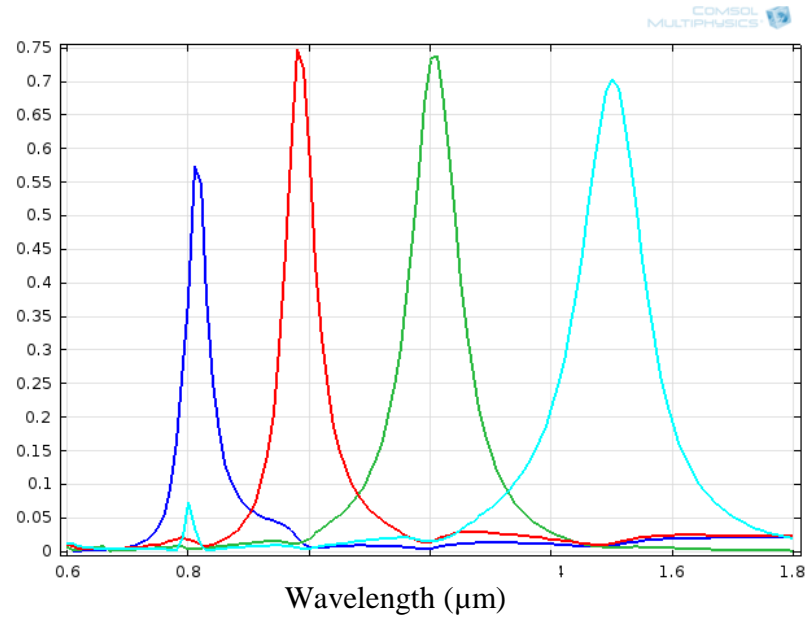

Fig7: Transmission spectra of four output channels of 3D plasmonic demultiplexer structure.

The finite element method (FEM) is used to simulate the transmission characteristics with different parameters of the nanocavity resonator. Figure (8) shows the transmission spectra for different refractive index and .According to equation: $\quad \lambda_{m} \frac{2 n_{e f f} L}{(m-\phi \mathrm{r} / \pi)}$ where $\lambda \mathrm{m}$ is resonance wavelength ,neff is effective refractive index,$L$ is length of nanocavity ,m is positive number and $\phi r=\phi 1+\phi 2, \phi 1$, and $\phi 2$ are, respectively, the phase shifts of the beam reflected at both ends of the nanocavity when increase the effective refractive index lead to increase resonance wavelength [2].The distribution electric field good an agreement of resonance wavelength channels as shown in the figure (9). 
The 2D demultiplexer based on plasmonic resonators has been discussed in many researches. contain on width and height only and neglected the third dimension. When the third dimension is considered, the structure will confine the light in three dimensions. In other word the structure can control the light in three dimensions, which can give more stability in light control. The fast development of plasmonics have greatly advanced understand to the abundant phenomena related to surface plamon polaritons (SPPs) and improved ability to manipulate light at the 3D nanometer scale. With tightly confined local field, SPPs can be transmitted in waveguides of subwavelength dimensions. Nanophotonic circuits built with plasmonic elements can be scaled down to dimensions compatible with semiconductor-based nanoelectronic circuits, which provides a potential solution for the next-generation information technology. The good feature of nanotechnology is nano scale size of component to increase density of component per unit area, and then enhance the performance of integrated circuit .

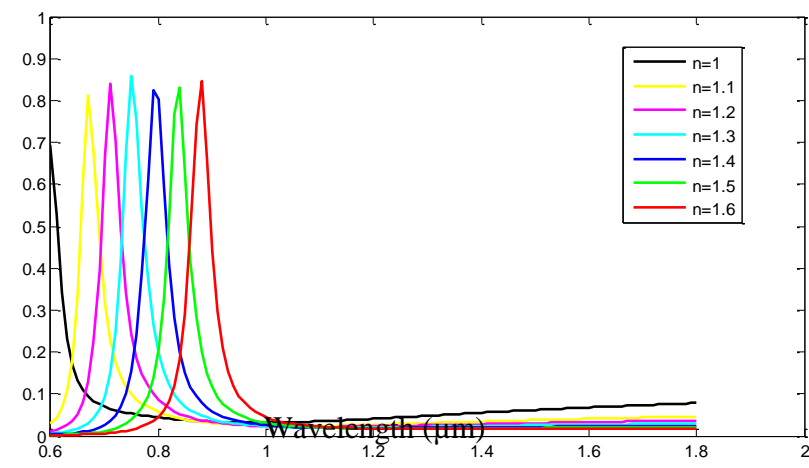

Fig 8: Effect refractive index on selective wavelength

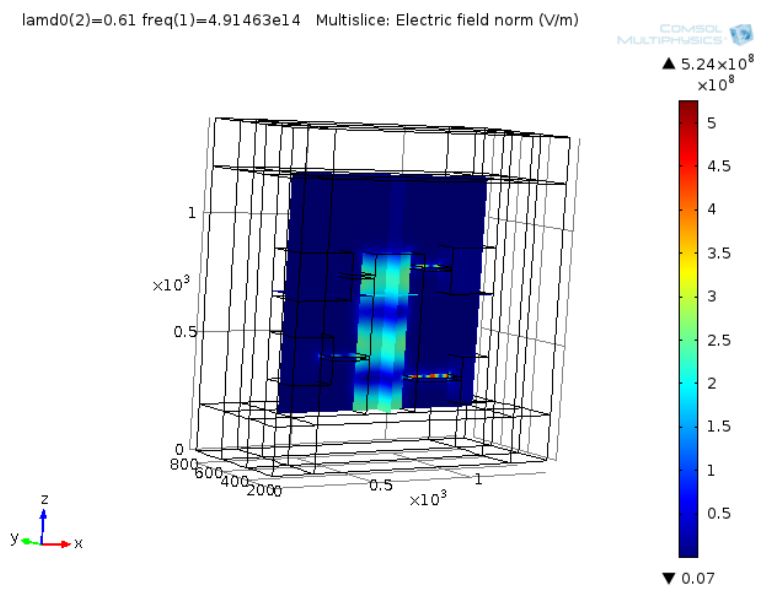

Fig9: The distribution electric field of four output channels of 3D plasmonic demultiplexer structure

\section{CONCLUSION}

In this paper, the resonance electric field of the light that propagated in the nanocavity of the $3 \mathrm{D}$ plasmonic demultiplexer is investigated, when changing the nanocavity length, width and thickness. From simulation results, it is found that the charge density is maximum at the resonance wavelength cause the electric field to be enhanced and decayed exponentially . . Has been conclude the four output channels selective plasmonic filter structure dependent on the distance between resonators that effect on interferencebetween channels. The required filtered wavelength can be conclude dependent on an appropriate selecting the length, width and thickness of nanocavity .Four output channels' structure is proposed to design a subwavelength plasmonic splitter and demultiplexer, based on four 3D nanocavity waveguides operating around of $810 \mathrm{~nm}$, $990 \mathrm{~nm}, 1210 \mathrm{~nm}$ and $1500 \mathrm{~nm}$ at special dimensions for resonators and ,there is possibility to change it by changing the length, width and thickness of resonators and then change the resonance wavelength. The FEM simulation results reveal that this new structure is suitable for wideband wavelengths demultiplexing. The transmission spectra at the outputs of the four channels. From the work conclude the small dimensions of proposed structures best choice for the fabrication of alloptical integrated devices and circuits for optical computing and communication, especially in WDM systems in the nanoscale

\section{REFERENCES}

[1] W. L. Barnes, A. Dereux, and T. W. Ebbesen, "Surface plasmon subwavelength optics," Nature 424(6950), 824-830 (2003).

[2] Q. Zhang, X. G. Huang, X. S. Lin, J. Tao, and X. P. Jin, "A subwavelength coupler-type MIM optical filter," Opt. Express 17(9), 7549-7554 (2009).

[3] C. Genet, and T. W. Ebbesen, "Light in tiny holes," Nature 445(7123), 39-46 (2007).

[4] C. Janke, J. G. Rivas, P. H. Bolivar, and H. Kurz, "All-optical switching of the transmission of electromagnetic radiation through subwavelength apertures," Opt. Lett. 30(18), 2357-2359 (2005).

[5] C. J. Min, P. Wang, C. C. Chen, Y. Deng, Y. H. Lu, H. Ming, T. Y. Ning, Y. L. Zhou, and G. Z. Yang, "Alloptical switching in subwavelength metallic grating structure containing nonlinear optical materials," Opt. Lett. 33(8), 869-871 (2008).

[6] G. A. Wurtz, R. Pollard, and A. V. Zayats, "Optical bistability in nonlinear surface-plasmon polaritonic crystals,” Phys. Rev. Lett. 97(5), 057402 (2006).

[7] B. Wang, and G. P. Wang, "Surface plasmon polariton propagation in nanoscale metal gap waveguides,” Opt. Lett. 29(17), 1992-1994 (2004).

[8] G. Veronis, and S. Fan, "Bends and splitters in metaldielectric-metal subwavelength plasmonic waveguides," Appl. Phys. Lett. 87(13), 131102 (2005).

[9] T. Nikolajsen, K. Leosson, and S. I. Bozhevolnyi, "Surface Plasmon polariton based modulators and switches operating at telecom wavelengths," Appl. Phys. Lett. 85(24), 5833 (2004).

[10] S. Randhawa, M. U. González, J. Renger, S. Enoch, and R. Quidant, "Design and properties of dielectric surfaceplasmon Bragg mirrors," Opt. Express 18(14), 14496-14510 (2010).

[11] S. Enoch, R. Quidant, and G. Badenes, "Optical sensing based on plasmon coupling in nanoparticle arrays," Opt. Express 12(15), 3422-3427 (2004). 
[12] J. Park, H. Kim, and B. Lee, "High order plasmonic Bragg reflection in the metal-insulator-metal waveguide Bragg grating," Opt. Express 16(1), 413425 (2008).

[13] Y. Gong, L. Wang, X. Hu, X. Li, and X. Liu, "Broadbandgap and low-sidelobe surface plasmon polariton reflector with Bragg-grating-based MIM waveguide," Opt. Express 17(16), 13727-13736 (2009).

[14] J. W. Mu, and W. P. Huang, "A Low-Loss Surface Plasmonic Bragg Grating," J. Lightwave Technol. 27(4), 436-439 (2009).

[15] X. S. Lin, and X. G. Huang, "Tooth-shaped plasmonic waveguide filters with nanometeric sizes," Opt. Lett. 33(23), 2874-2876 (2008).

[16] J. Tao, X. G. Huang, X. Lin, Q. Zhang, and X. Jin, “A narrow-band subwavelength plasmonic waveguide filter with asymmetrical multiple-teeth-shaped structure," Opt. Express 17(16), 13989-13994 (2009).

[17] S. S. Xiao, L. Liu, and M. Qiu, "Resonator channel drop filters in a plasmon-polaritons metal," Opt. Express 14(7), 2932-2937 (2006).
[18] A. Hosseini, and Y. Massoud, "Nanoscale surface Plasmon based resonator using rectangular geometry," Appl. Phys. Lett. 90(18), 181102 (2007).

[19] T. B. Wang, X. W. Wen, C. P. Yin, and H. Z. Wang, "The transmission characteristics of surface plasmon polaritons in ring resonator," Opt. Express 17(26), 24096-24101 (2009).

[20] A. Noual, A. Akjouj, Y. Pennec, J. Gillet, and B. Djafari-Rouhani, "Modeling of two-dimensional nanoscale Ybent plasmonic waveguides with cavities for demultiplexing of the telecommunication wavelengths," N. J. Phys. 11(10), 103020 (2009).

[21] A. Taflove, and S. C. Hagness, "Computational Electrodynamics: The Finite-Difference TimeDomain Method," 2nd ed. 2000 (Artech House, Boston).

[22] P.B. Johnson and R.W. Christy "Optical Constant of the Nobel Metals" Physical Review B volume 6, number 1215 December 1972.

[23] CHEN Zhao.YU Li,WANG Lu-Lu, ZHAO Yu-Fang, DAN Gao-Yan, XIAO Jing-Hua" High-Resolution Compact Plasmonic Wavelength Demultiplexers Based on Cascading Square Resonators" Vol. 30, No. 5 (2013) 054212. 